\title{
Striatal Neuroinflammation Promotes Parkinsonism in Rats
}

\author{
Dong-Young Choi ${ }^{1}$, Mei Liu ${ }^{1}$, Randy L. Hunter ${ }^{1}$, Wayne A. Cass ${ }^{1}$, Jignesh D. Pandya ${ }^{2}$, Patrick G. \\ Sullivan ${ }^{1,2}$, Eun-Joo Shin ${ }^{3}$, Hyoung-Chun Kim ${ }^{3}$, Don M. Gash ${ }^{1}$, Guoying Bing ${ }^{1 *}$
}

1 Department of Anatomy and Neurobiology, University of Kentucky, Lexington, Kentucky, United States of America, 2 Spinal Cord and Brain Injury Research Center, University of Kentucky, Lexington, Kentucky, United States of America, 3 Neuropsychopharmacology and Toxicology Program, College of Pharmacy, Kangwon National University, Chunchon, South Korea

\begin{abstract}
Background: Sporadic Parkinson's disease (PD) is a progressive neurodegenerative disorder with unknown cause, but it has been suggested that neuroinflammation may play a role in pathogenesis of the disease. Neuroinflammatory component in process of PD neurodegeneration was proposed by postmortem, epidemiological and animal model studies. However, it remains unclear how neuroinflammatory factors contribute to dopaminergic neuronal death in PD.

Findings: In this study, we analyzed the relationship among inducible nitric oxide synthase (iNOS)-derived NO, mitochondrial dysfunction and dopaminergic neurodegeneration to examine the possibility that microglial neuroinflammation may induce dopaminergic neuronal loss in the substantia nigra. Unilateral injection of lipopolysaccharide (LPS) into the striatum of rat was followed by immunocytochemical, histological, neurochemical and biochemical analyses. In addition, behavioral assessments including cylinder test and amphetamine-induced rotational behavior test were employed to validate ipsilateral damage to the dopamine nigrostriatal pathway. LPS injection caused progressive degeneration of the dopamine nigrostriatal system, which was accompanied by motor impairments including asymmetric usage of forelimbs and amphetamine-induced turning behavior in animals. Interestingly, some of the remaining nigral dopaminergic neurons had intracytoplasmic accumulation of $\alpha$-synuclein and ubiquitin. Furthermore, defect in the mitochondrial respiratory chain, and extensive S-nitrosylation/nitration of mitochondrial complex I were detected prior to the dopaminergic neuronal loss. The mitochondrial injury was prevented by treatment with L- $\mathrm{N}^{6}$-(l-iminoethyl)-lysine, an iNOS inhibitor, suggesting that iNOS-derived NO is associated with the mitochondrial impairment.
\end{abstract}

Conclusions: These results implicate neuroinflammation-induced S-nitrosylation/nitration of mitochondrial complex I in mitochondrial malfunction and subsequent degeneration of the nigral dopamine neurons.

Citation: Choi D-Y, Liu M, Hunter RL, Cass WA, Pandya JD, et al. (2009) Striatal Neuroinflammation Promotes Parkinsonism in Rats. PLoS ONE 4(5): e5482. doi:10.1371/journal.pone.0005482

Editor: Howard E. Gendelman, University of Nebraska, United States of America

Received January 12, 2009; Accepted April 15, 2009; Published May 8, 2009

Copyright: $@ 2009$ Choi et al. This is an open-access article distributed under the terms of the Creative Commons Attribution License, which permits unrestricted use, distribution, and reproduction in any medium, provided the original author and source are credited.

Funding: This work was supported by grants from the National Institute on Aging, the National Institute of Neurological Disorders and Stroke: NS39345 and NS044157 (to GYB), and Brain Research Center grant from the 21st Century Frontier Research Program funded by the Ministry of Science and Technology, Republic of Korea (to HCK). The funders had no role in study design, data collection and analysis, decision to publish, or preparation of the manuscript.

Competing Interests: The authors have declared that no competing interests exist.

* E-mail: gbing@uky.edu

\section{Introduction}

Microglial activation is a pathological hallmark of neurodegenerative diseases including Parkinson's disease (PD) [1]. Microgliosis is a normal response in the damaged CNS, which can promote sprouting of injured neurons by providing neurotrophic factors [2]. On the other hand, the activated microglia may be destructive to neurons by releasing inflammatory molecules such as nitric oxide (NO), and cytokines [3,4]. While the role of activated microglia in the parkinsonian brain is controversial, the observation of persistent microgliosis in the substantia nigra of parkinsonian patients, and 1-methyl-4-phenyl-1,2,3,6-tetrahydropyridine-exposed humans and animals has led to a postulation that the chronic inflammatory response might contribute to loss of the dopamine neurons [5-8]. A study using positron emission tomography with $\left[{ }^{11} \mathrm{C}\right](R)$-PK 11195 , a radiotracer for activated microglia and $\left[{ }^{11} \mathrm{C}\right] \mathrm{CFT}$, a dopamine transporter marker reported that microglial activation level in the midbrain is positively correlated with severity of motor impairment and dopaminergic terminal loss in the putamen of early PD patients, suggesting microglial neuroinflammatory response may be related to progressive neurodegeneration of the disease [9]. In line with the observation, an epidemiological study illustrated that the risk of developing PD is significantly reduced by regular use of nonsteroidal anti-inflammatory drugs such as ibuprofen [10]. In addition, reactive microglia express increased levels of inflammatory enzymes including inducible nitric oxide synthase (iNOS) and cyclooxygenase-2 in the substantia nigra of PD brain [11]. Importantly, these enzymes appear to play a pivotal role in the loss of dopaminergic neurons in animal models of PD [12-14].

iNOS is expressed in microglia during neuroinflammation and produces excessive amount of $\mathrm{NO}$, which can cause death of neurons [15]. The gaseous molecule released at extramitochondrial sites can diffuse into the mitochondria and react with 
mitochondria-derived superoxide anion to form peroxynitrite, which accounts for much of the disruption of mitochondrial function [16]. Moreover, peroxynitrite inhibits the activity of manganese superoxide dismutase, which could enhance formation of superoxide anion and peroxynitrite [17]. It has been documented that NO and its metabolite peroxynitrite inhibit mitochondrial respiration via S-nitrosylation and nitration of complex I, respectively [18]. Impairment in the mitochondrial electron transport chain has been suggested to be a critical factor in the pathogenesis of PD, as defect in mitochondrial complex I is consistently detected in PD patients [19,20] and mitochondrial toxins induces parkinsonism [21-23]. The dopaminergic neurons can be particularly susceptible to NO neurotoxicity because dopamine potentiates NO-induced impairment in mitochondrial respiration [24]. Furthermore, metabolism of dopamine by monoamine oxidase, a mitochondrial enzyme may enhance the mitochondrial lesion in the dopamine-producing neurons [25].

We and other groups have demonstrated that intranigral [2628] or intrapalidal [29] lipopolysaccharide (LPS) induces death of the nigral dopaminergic neurons through microglial activation. However, these animal models did not recapitulate some cardinal features of PD such as progressive dopaminergic neurodegeneration, Lewy body-like intracytoplasmic inclusion and parkinsonian behavioral impairments. Furthermore, underlying mechanism(s) by which microglial neuroinflammation kills the nigral dopaminergic neurons has not been fully delineated. This study was designed to demonstrate striatal neuroinflammation-induced dopaminergic neurodegeneration and explore how neuroinflammation mediates the loss of dopamine-producing neurons. Here, we report that LPS-induced striatal inflammation causes the impairment of the mitochondrial respiratory chain in both the substantia nigra and striatum, which was followed by progressive degeneration of the dopamine nigrostriatal pathway, behavioral impairment, and accumulation of $\alpha$-synuclein and ubiquitin in the remaining nigral dopaminergic neurons. In addition, our results indicate that NO produced by iNOS is related to S-nitrosylation/ nitration of mitochondrial complex I which might lead to mitochondrial dysfunction and the subsequent dopaminergic neurodegeneration.

\section{Methods}

\section{Animals and surgery}

All animal experiments were performed according to the NIH Guide for the Care and Use of Laboratory Animals and were approved by the University of Kentucky Institutional Animal Care and Use Committee. 3 month-old male Sprague-Dawley rats (Harlan, Indianapolis, IN) were housed under a twelve hour light-dark cycle with free access to food and water in the Division of Lab Animal Resources at the University of Kentucky. For the injection of LPS (Salmonella minnesota; Sigma-Aldrich, St Louis, MO), rats were deeply anesthetized with sodium pentobarbital ( $50 \mathrm{mg} / \mathrm{kg}$ i.p.) and were positioned in a stereotaxic frame (Stoelting Co., Wood Dale, IL) with the incisor bar at the level of the ear. LPS dissolved in saline $(2.5 \mu \mathrm{g} / \mu \mathrm{l})$ was injected into the right striatum $(3 \mu \mathrm{l} /$ site $)$ using the following coordinates (in $\mathrm{mm}$ ): site 1 , anteroposterior (AP) 1.0, mediolateral (ML) 2.0, dorsoventral (DV) -5.5; site 2, AP 1.0, ML 3.5, DV -6.0; site 3, AP -0.5, ML 2.5, DV - 5.0; site $4, \mathrm{AP}-0.5$, ML 4.0, DV -6.5. Saline was injected into the left striatum with parallel coordinates. Placebo animals received saline injections into the right and left striatum with the same regimen. After surgery, animals were kept on heating pad until recovery from surgery and subcutaneous saline was given for aid in postoperative recovery. In order to investigate the detrimental effect of
iNOS on the mitochondria, animals were treated with $\mathrm{L}-\mathrm{N}^{6}$-(1iminoethyl)-lysine (L-NIL, $5 \mathrm{mg} / \mathrm{ml} / \mathrm{kg}$, i.p., Cayman Chemical, Ann Arbor, MI) or its vehicle (sterilized saline) $20 \mathrm{~min}$ before, and one day and two days after LPS injection.

\section{Histopathology}

Animals were perfused with $4 \%$ paraformaldehyde three days, one week, two weeks, and four weeks after LPS injections $(\mathrm{n}=5$ 6/group) for immunohistochemistry and histopathological examinations. Coronal sections $(30 \mu \mathrm{m})$ were cut through the entire brain using a sliding microtome. Immunohistochemistry, with slight modifications, was performed as previously described [28]. Briefly, every sixth section from the region containing the striatum or substantia nigra was blocked with $5 \%$ bovine serum albumin for $1 \mathrm{hr}$ at room temperature. The brain sections were immunolabeled with antibody to tyrosine hydroxylase ( $\mathrm{TH}$, Calbiochem, San Diego, CA, 1:3,000), MHC class II (OX-6, Serotec, Raleigh, NC, 1:1,000) or dopamine- and cAMP-regulated phosphoprotein polypeptide (DARPP-32, Chemicon, Temecula, CA, 1:1,000, $24 \mathrm{hrs}$ at $4^{\circ} \mathrm{C}$ ). After multiple washings, sections were incubated with species-appropriate secondary antibody (1:1,000; Vector Laboratories, Burlingame, CA). The final antigen-antibody complex was visualized using an Avidin-biotin complex (ABC kit, Vector laboratories) method and 3,3'-diaminobenzidine tetrachloride as a chromagen (Sigma-Aldrich).

Colocalization of $\mathrm{TH}$ with $\alpha$-synuclein, or ubiquitin was assessed as previously described [29]. Briefly, substantia nigracontaining sections were incubated with primary polyclonal antibody against $\alpha$-synuclein (1:500; Sigma-Aldrich) or ubiquitin (1:1,000; Sigma-Aldrich) overnight at $4^{\circ} \mathrm{C}$. The sections were incubated in Alexa Fluor 488 goat anti-rabbit secondary antibody (1:1,000; Molecular Probes Inc., Eugene, OR) for $1 \mathrm{hr}$, at room temperature. The sections were subsequently incubated with mouse anti-TH primary antibody (1:1,000; Calbiochem) overnight at $4^{\circ} \mathrm{C}$ followed by incubation for $1 \mathrm{hr}$ in Alexa Fluor 568 goat anti-mouse IgG secondary antibody (1:1,000; Molecular Probes Inc.) at room temperature. The fluorescent specimens were first assessed by an Axioplan 2 microscope (Carl Zeiss, Inc.) and images were acquired by using an Axiocam digital camera connected to a computer equipped with Axiovision 3.0 software (Carl Zeiss, Inc.). Fluorescent preparations were also examined using the Leica TCS SP laser scanning confocal imaging system (Leica Microsystems, Inc., Buffalo, NY). Images were viewed on a Leica DM RXE upright microscope. Photomicrographs were captured simultaneously for both fluorophores [Alexa Fluor 488 (green) and Alexa Fluor 568 (red)] by using argon and krypton lasers, respectively. Regions exhibiting colocalization of the red and green emitters produced yellow fluorescence.

Another set of sections were rinsed in PBS and then incubated in PBS containing proteinase $\mathrm{K}(20 \mu \mathrm{g} / \mathrm{ml})$ for $30 \mathrm{mins}$ at room temperature. After multiple washings in PBS, these sections were subjected to immunostaining with antibodies to $\alpha$-synuclein (1:5,000, Chemicon, Temecula, CA) or ubiquitin (1:5,000, Sigma-Aldrich).

Standard Nissl staining was performed to assess neuronal morphology and population in the substantia nigra. Degenerative neuronal cell bodies and neurites in the substantia nigra and striatum were visualized using the FD Neurosilver kit (FD NeuroTechnologies, Ellicott City, MD), which was performed according to the manufacturer's protocol. Photomicrographs were taken by using an Axiocam digital camera connected to a computer equipped with Axiovision 3.0 software (Carl Zeiss, Inc., Thornwood, NY). 


\section{Quantification of immunohistochemistry}

The number of $\mathrm{TH}$-positive neurons in the substantia nigra was determined using the computerized optical fractionator method of the Bioquant system (Memphis, TN, USA), which was described previously [30,31]. Striatal TH-positive fiber density was measured as previously described [32]. Briefly, striatum-containing sections were picked at seven rostrocaudal levels, covering the entire head and tail of the striatum, corresponding to: (i) $\mathrm{AP}+1.60$; (ii) $\mathrm{AP}+1.00$; (iii) $\mathrm{AP}+0.20$; (iv) $\mathrm{AP}-0.30$; (v) $\mathrm{AP}-0.90$; (vi) $\mathrm{AP}$ -1.40 and (vii) AP -2.10 relative to bregma. TH-positive fiber density was measured by using Bioquant Image Analysis software. Each value was corrected for non-specific background by subtracting the optical density of corpus callosum.

\section{HPLC analysis for levels of striatal neurochemicals}

Animals were killed four weeks following LPS challenge for determination of striatal dopamine, serotonin and their metabolites levels ( $\mathrm{n}=7$ /group). Levels of striatal dopamine, 3,4dihydroxyphenylacetic acid (DOPAG), homovanillic acid (HVA), serotonin, and 5-hydroxyindole acetic acid (5-HIAA) were determined by using HPLC. Analyses were performed as previously described [33]. Briefly, tissue samples were sonicated in $300 \mu \mathrm{l}$ of cold $0.1 \mathrm{M}$ perchloric acid containing dihydroxybenzylamine as an internal standard. The supernatant was separated by centrifugation at $12,000 \mathrm{~g}$ for five minutes, and was transferred to Millipore Ultrafree centrifugal filters (pore size, $0.22 \mu \mathrm{m})$, and then were spun at $12,000 \mathrm{~g}$ for one minute. The filtrate was diluted with HPLC mobile phase, and $50 \mu \mathrm{l}$ was injected onto the HPLC column. The HPLC system consisted of a Beckman Model 507 autoinjector, a Beckman Model 118 pump, and an ESA Model 5200A Coulochem II electrochemical detector with a Model 5011 dual-detector analytical cell (detector 1 set at $+350 \mathrm{mV}$ and detector 2 set at $-300 \mathrm{mV}$ ). An ESA Hypersil ODS $3 \mu \mathrm{m}$ particle $\mathrm{C}_{18}$ column $(80 \times 4.6 \mathrm{~mm})$ was used for separations. Flow rate was $1.4 \mathrm{ml} / \mathrm{min}$ and the mobile phase was a $\mathrm{pH} 4.1,0.17 \mathrm{M}$ citrate-acetate buffer (containing $5 \mathrm{mg} / \mathrm{l}$ EDTA, 70-80 mg/l octanesulfonic acid, and 7-8\% methanol). Chromatograms were recorded from both detectors using two dual-channel strip chart recorders. Retention times of standards were used to identify peaks, and peak heights were used to calculate recovery of internal standard and amount of dopamine and metabolites. Tissue monoamine concentrations of dopamine, DOPAC, HVA, serotonin, and 5-HIAA are expressed as $\mathrm{ng} / \mathrm{mg}$ wet tissue.

\section{Behavioral assessment}

Test for amphetamine-induced rotational behavior was performed four weeks following LPS injection ( $n=5-6$ /group). For the test, animals were placed in a hemispherical bowl immediately after receiving $5 \mathrm{mg} / \mathrm{kg}$ amphetamine injection (i.p.). The behavior of each animal was monitored through an automated video-tracking system, and the number of ipsilateral $360^{\circ}$ turns was determined for 90 minutes per animal.

Cylinder test is a motor assessment of forelimb asymmetry, and was performed as described previously with slight modifications [34]. Briefly, rats were individually put into a glass cylinder (20 cm diameter, $34 \mathrm{~cm}$ height) and were video recorded until they touched the cylinder wall with their forelimbs 20 times. The recordings were analyzed by an investigator who was not aware of the identity of the rats. The data are presented as the asymmetric score calculated by the following formula: (Right touch-Left touch) $/($ Right touch+Left touch+Both touch $)(\mathrm{n}=5-$ 6/group).

\section{Mitochondrial isolation and measurement of their respiration}

Mitochondria were isolated using discontinuous Ficoll gradient and differential centrifugation with nitrogen disruption, and respiration was assessed as previously described with slight modifications [35]. Briefly, rats were killed three days after LPS injections and the nigral and striatal tissues were immediately and carefully dissected. Two unilaterally injected striata or four nigra, ipsilateral to the injections, had to be pooled to obtain one sample ( $\mathrm{n}=6-9$ /group). The tissues were homogenized with ice-cold isolation buffer (pH 7.2, $215 \mathrm{mM}$ mannitol, $75 \mathrm{mM}$ sucrose, $0.1 \%$ bovine serum albumin, $20 \mathrm{mM}$ HEPES, $1 \mathrm{mM}$ EGTA) and the crude mitochondrial fraction was obtained by differential centrifugation and nitrogen disruption. Further purification was performed using a Ficoll gradient and differential centrifugation. Mitochondrial respiratory rates were measured using a Clark-type electrode in a continuously stirred and sealed thermostatically controlled chamber (Oxytherm System, Hansatech Instruments Ltd., King's Lynn, Norfolk, UK) maintained at $37^{\circ}$ C. 25 to $40 \mathrm{mg}$ of isolated mitochondrial protein was placed in the chamber containing $250 \mathrm{ml}$ of KCl-based respiration buffer $(125 \mathrm{mM} \mathrm{KCl}$, $2 \mathrm{mM} \mathrm{MgCl}_{2}, 2.5 \mathrm{mM} \mathrm{KH}_{2} \mathrm{PO}_{4}, 0.1 \%$ BSA, $20 \mathrm{mM}$ HEPES at $\mathrm{pH}$ 7.2) and allowed to equilibrate for $1 \mathrm{~min}$. State II respiration was initiated by the addition of complex I substrates, pyruvate $(5 \mathrm{mM})$ and malate $(2.5 \mathrm{mM})$, to monitor. Then ADP $(150 \mathrm{mM})$ were added to the mitochondria to assess state III respiratory rate for 2 mins, followed by the addition of oligomycin $(2 \mathrm{mM})$ to monitor state IV respiration rate for an additional 2 mins. For the measurement of uncoupled respiratory rate, FCGP $(2 \mathrm{mM})$ was added to the mitochondria in the chamber and oxygen consumption rate was measured for another 2 mins. This was followed by the addition of succinate $(10 \mathrm{mM})$ to monitor complex II-driven respiration. The results were the rates of oxygen consumption in nanoatoms of oxygen $/ \mathrm{min} / \mathrm{mg}$ protein and presented as percentage of control.

\section{Assessment of mitochondrial protein S-nitrosylation/ nitration}

Approximately, $200 \mu \mathrm{g}$ of mitochondrial protein was solubilized in RIPA buffer containing 1\% lauryl maltoside (Sigma-Aldrich) and centrifuged at 20,000g for $30 \mathrm{~min}$ to collect the supernatant. The mitochondrial solution was incubated at $4{ }^{\circ} \mathrm{C}$ for $24 \mathrm{hr}$ with $15 \mu \mathrm{l}$ of agarose beads, irreversibly cross-linked to complex I specific antibodies (MitoSciences, Eugene, OR). The beads were collected by centrifugation and washed three times with PBS containing $0.05 \%$ laruryl maltoside. The beads were then resuspended in $50 \mu \mathrm{l}$ of sample loading buffer with non-reducing condition and agitated for $10 \mathrm{~min}$. After gentle centrifugation, $10 \mu \mathrm{l}$ of the supernatant was loaded into a $12 \%$ SDS-PAGE gel and proteins were resolved. The proteins were transferred to PVDF membrane and detected by incubation with a polyclonal antibody against 3-nitrotyrosine (Upstate Biotechnology, Lake Placid, NY) or S-nitrosylcystein (Sigma-Aldrich), which was followed by horseradish peroxidase labeled goat anti-rabbit IgG (Sigma-Aldrich). Chemiluminescent detection with the ECL Plus kit and exposure to x-ray film was performed, and protein levels were quantified by the Scion Image software (Scion Corporation, Frederick, MD) ( $\mathrm{n}=4$ /group).

\section{Western blot analysis}

Animals were sacrificed six hours, one day, and three days after LPS or saline injections. The striatum and substantia nigra were dissected out on ice. Tissues were homogenized in ice-cold lysis 
A

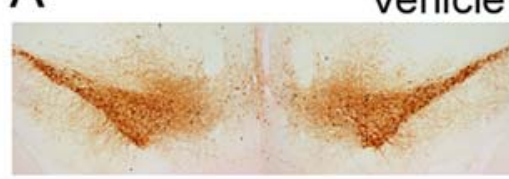

2 weeks

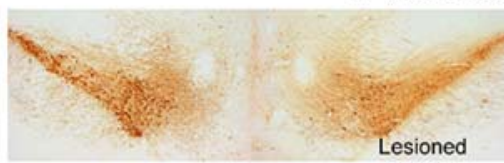

B

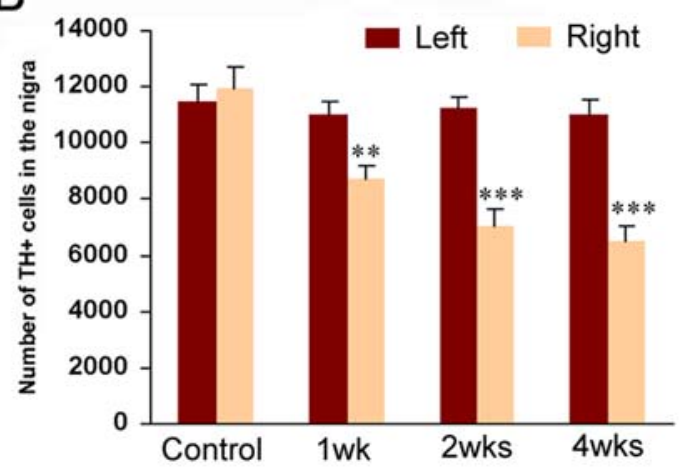

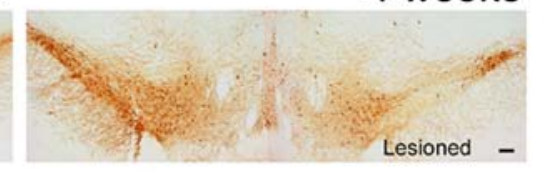

1 week

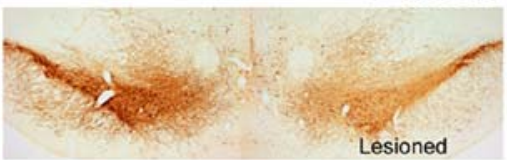

4 weeks

\section{(}
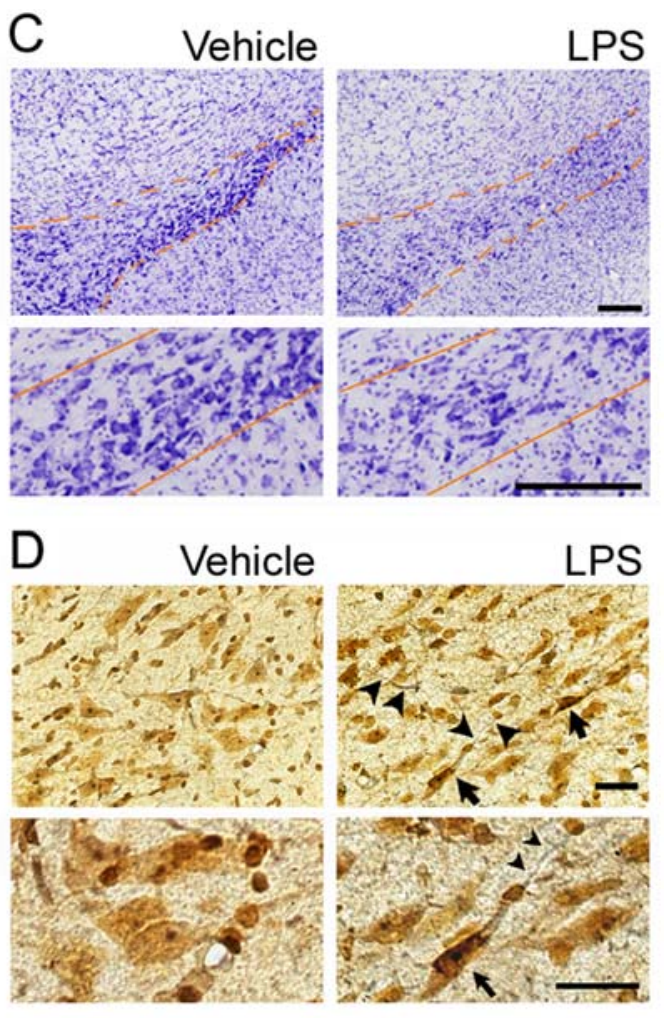

Figure 1. Progressive degeneration of the nigral dopaminergic neurons after intrastriatal LPS. (A) Representative TH immunostaining of coronal midbrain sections demonstrates that the numbers of TH-positive neurons and fibers in the substantia nigra pars compacta are gradually reduced by intrastriatal LPS injection. Note that TH-positive neurons in the medial substantia nigra pars compacta and ventral tegmental area are spared; scale bar: $200 \mu \mathrm{m}$. (B) Stereological cell counts of the TH-positive neurons in the substantia nigra pars compacta $(n=5-6 / g r o u p, * * 0<0.01$, *** $p<0.001$ ). (C) The substantia nigra pars compacta is outlined with an orange dashed line (top). High magnification image of Nissl stainings suggest loss of the nigral dopaminergic neurons, at four weeks following LPS injection (bottom); scale bar: $200 \mu \mathrm{m}$. (D) Silver staining is hardly seen in the substantia nigra ipsilateral to vehicle treatment. However, abundant silver grain-deposits are observed in the neurons (arrows) and fibers (arrow heads) in the substantia nigra ipsilateral to the intrastriatal LPS injections, indicating there is ongoing neurodegenerative process in the region. Scale bar: $20 \mu \mathrm{m}$.

doi:10.1371/journal.pone.0005482.g001

buffer and centrifuged $(10,600 \mathrm{~g})$ to collect the supernatant. All of the samples were kept at $-70^{\circ} \mathrm{C}$ until they were used for analysis. Next, $15 \mu \mathrm{g}$ of protein from each sample was aliquoted, and after addition of loading buffer, the protein was loaded and resolved using a $12 \%$ SDS-PAGE gel. Protein on the gel was transferred to a nitrocellulose membrane, which was blocked in 5\% fat-free milk at $4^{\circ} \mathrm{C}$ overnight. The membrane was incubated in primary antibodies to iNOS (1:1,000, Upstate Biotechnology) or DARPP$32(1: 4,000)$ at room temperature for one hour. Then, the membrane was rinsed with tris-buffered saline three times for 15 min each before incubation in secondary antibody (goat antirabbit, 1:2,000; Sigma-Aldrich) for one hour. This was followed by treatment with the ECL chemiluminescent reagents (Amersham Biosciences, Piscataway, NJ) and exposure to film. A density measurement for each band was performed with the Scion Image software (Scion Corporation). Background values from an equivalent area near each lane were subtracted from each band to calculate mean band density and iNOS or DARPP-32 immunoreactivity was normalized by density of $\beta$-actin bands in the same membrane to correct loading error ( $n=3-4$ /group).

\section{Statistical analysis}

Animals were randomly grouped, and tests of variance homogeneity, normality, and distribution were performed to ensure that the assumptions required for standard parametric analysis of variance (ANOVA) were satisfied. The Systat 10 software (SPSS Inc., Chicago, IL) was used to perform statistical analyses by using the linear correlation unpaired test, Student's $t$ test or ANOVA followed by a protected least significant differences post hoc test only following a positive $\mathrm{F}$ test result. Statistical significance was set at $\mathrm{p}<0.05$. The linear correlation unpaired test was performed to analyze correlation between loss of nigral TH-positive cells and time. The ANOVA was used for analysis of the stereological cell counts, mitochondrial respiration data, RNase protection assay, and western blot. The Student's $t$ test was used to analyze HPLC and behavioral data. Data are expressed as means \pm s.e.m.

\section{Results}

\section{Progressive degeneration of the dopamine nigrostriatal system}

To characterize and quantify the loss of dopaminergic neurons in the midbrain after LPS injection, immunostaining with an antibody against $\mathrm{TH}$ and non-biased stereological estimation of the TH-positive neurons in the substantia nigra were performed. Abundant TH-positive cell bodies and fibers existed in the substantia nigra and ventral tegmental area of placebo and 
A

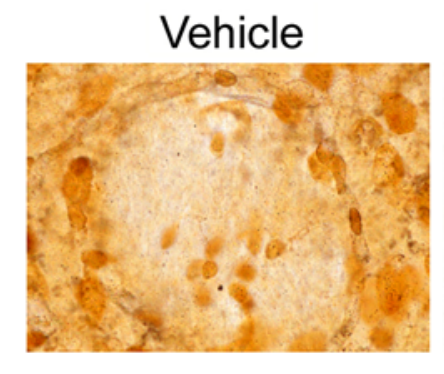

B
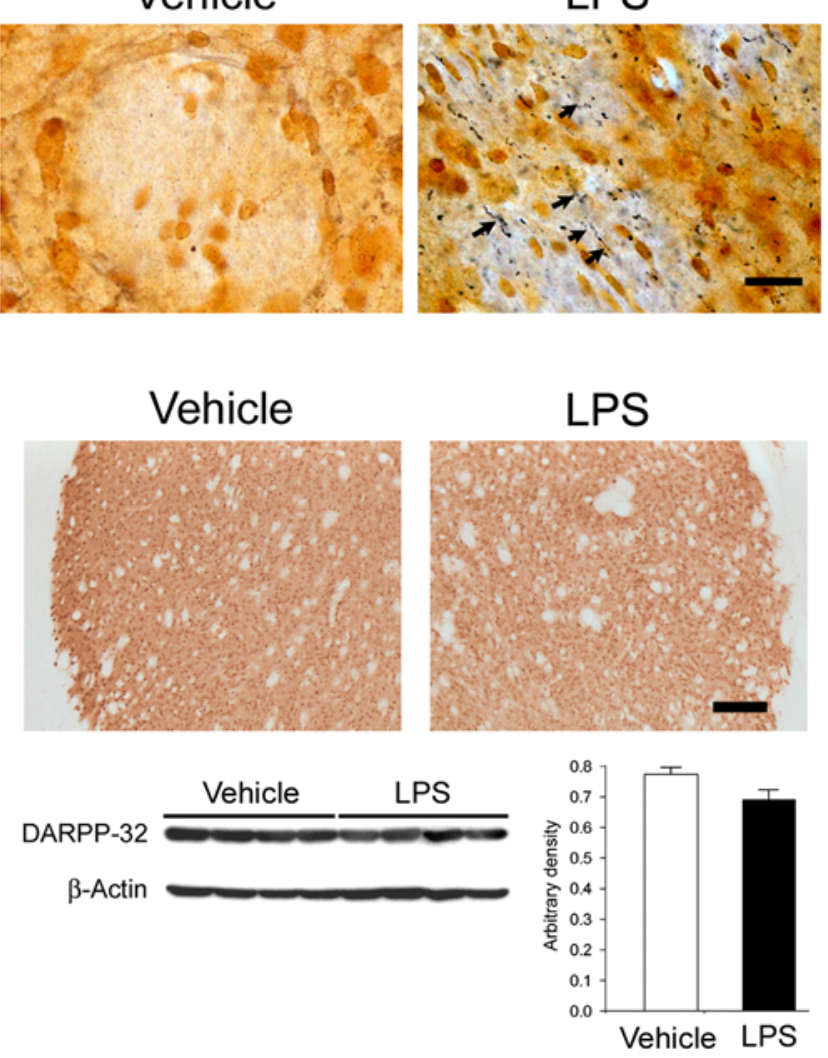
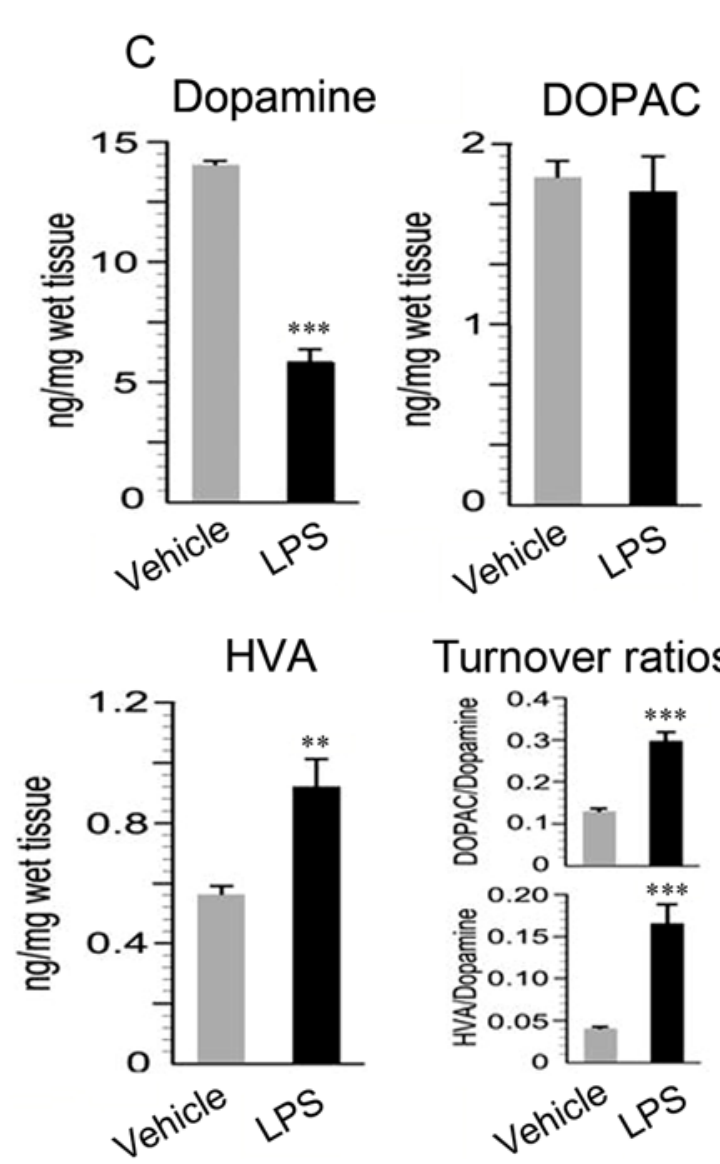

Figure 2. Axonal terminal degeneration in the striatum following intrastriatal LPS. (A) Silver staining reveals that there is no silver-positive stained fibers in the vehicle treated striatum while an abundance of silver grain-deposits are observed in the LPS injected striatum, suggesting the degeneration of axonal fibers (arrows). Scale bar: $20 \mu \mathrm{m}$. (B) Immunostaining for DARPP-32 shows that the GABAergic neurons are intact following LPS injections. Western blot for DARPP-32 and its quantification indicate that there is no significant alteration in the expression of DARPP-32 after LPS challenge. Scale bar: $200 \mu \mathrm{m}$. (C) HPLC analysis shows that intrastriatal LPS injection depletes $58 \%$ of the striatal dopamine relative to control at four weeks. The DOPAC level is not affected; however, HVA is significantly increased. The turnover ratios of DOPAC/dopamine and HVA/dopamine are dramatically increased $\left(n=7 /\right.$ group; $\left.{ }^{* *} p<0.01,{ }^{* * *} p<0.001\right)$.

doi:10.1371/journal.pone.0005482.g002

saline-treated side. In contrast, the number of TH-positive cells and fibers progressively decreased in the substantia nigra ipsilateral to the LPS injection, while TH-positive neurons in the ventral tegmental area were spared (Fig. 1A). Stereological estimation of the spared nigral TH-positive cells showed a progressive and significant loss of the cells: $21 \%$ at one week $\left(p=8 \times 10^{-3}\right), 38 \%$ at two weeks $\left(p=1 \times 10^{-5}\right)$, and $41 \%$ at four weeks $\left(p=2 \times 10^{-7}\right.$; Fig. 1B). Stereological analysis indicated that the number of TH-positive cells in the ventral tegmental area was not significantly affected by LPS injection $(98.9 \pm 2.9 \%$ at one week, $100.2 \pm 7.1 \%$ at two weeks and $97.3 \pm 7.4 \%$ at four weeks vs. control). Nissl staining of adjacent sections showed fewer large neurons in the substantia nigra, consistent with the loss of dopaminergic neurons four weeks after LPS injection (Fig. 1C). To detect ongoing degenerative events of the nigrostriatal dopaminergic system, silver staining was performed. No nigral degeneration was detected in the substantia nigra ipsilateral to the vehicleinjected striatum (Fig. 1D). However, nigral neurons with silver deposits in their cell bodies or fibers were observed in the substantia nigra ipsilateral to the LPS-injected side (Fig. 1D). In addition to the loss of dopaminergic cell bodies in the substantia nigra, silver staining of striatal sections revealed that axon terminals were undergoing degeneration by LPS-induced inflammation, as shown by dense staining with silver grains (Fig. 2A). Density of striatal TH-positive fiber was also significantly reduced four weeks after LPS injection (Fig. S1, 15\%, p=0.001). In contrast, the immunostaining for DARPP-32 indicated that the population of gamma-aminobutyric acid (GABA) neurons in the striatum did not seem to be affected by the LPS infusions (Fig. 2B, top panel). In agreement with the immunostaining, expression level of DARPP-32 was not significantly altered by LPS challenge as determined by Western blot analysis (Fig. 2B, bottom panel). Consistent with the degeneration of the nigrostriatal system, the level of striatal dopamine significantly declined to $42 \%$ of the control level, four weeks after LPS injection $\left(p=1 \times 10^{-9}\right)$. The turnover ratio of DOPAC $\left(\mathrm{p}=1 \times 10^{-6}\right)$ or HVA $\left(\mathrm{p}=1 \times 10^{-5}\right)$ to dopamine was significantly increased, as occurs in PD [36]. There was also a significant increase in HVA level $(p=0.004)$ but not DOPAC following LPS (Fig. 2C), suggesting an alteration in dopamine metabolism. Serotonin but not its primary metabolite, 5 -HIAA was also significantly decreased $(\mathrm{p}=0.0002)$ leading to a significant increase in the turnover ratio (5-HIAA/serotonin; $\mathrm{p}=2 \times 10^{-5}$ ) (Fig. S2), which may indicate that serotonergic neurons were affected by LPS infusion. 
A
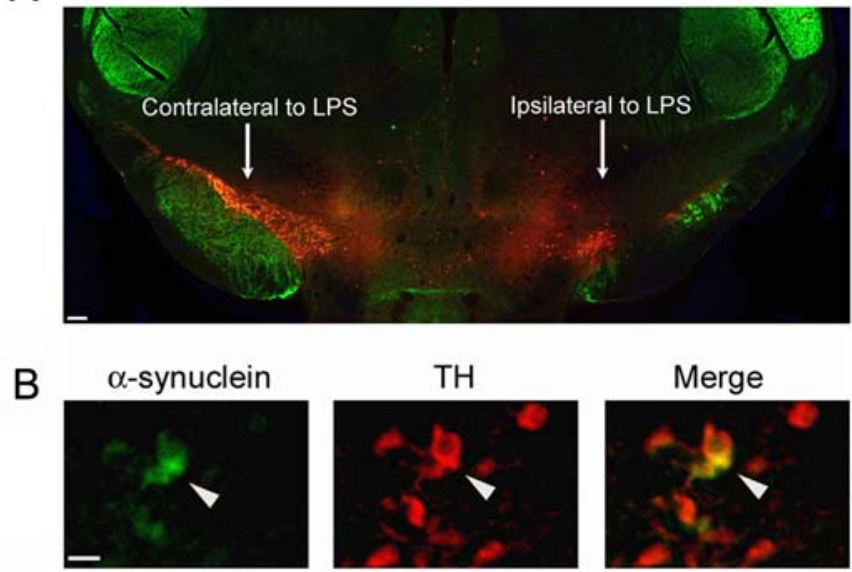

C

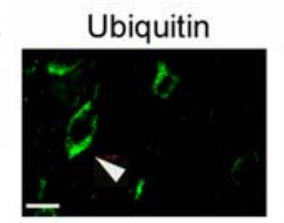

TH

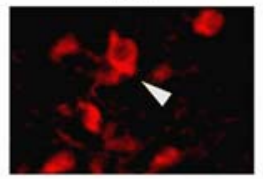

$\mathrm{TH}$

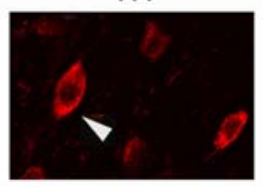

D

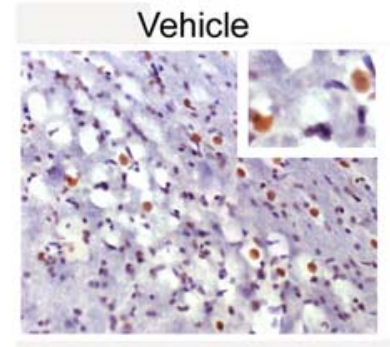

E

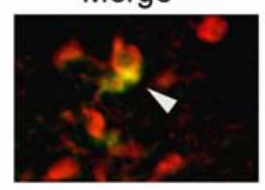

Merge

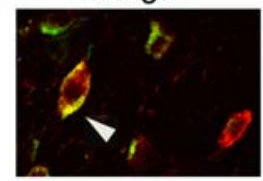

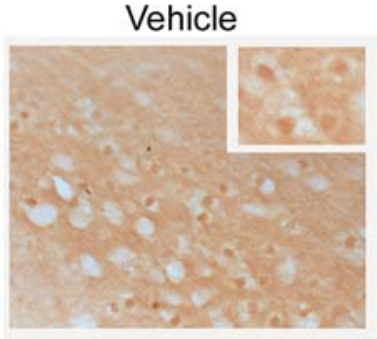

LPS

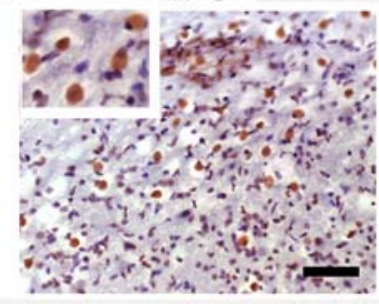

LPS

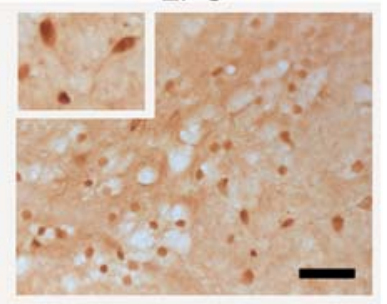

Figure 3. Cytoplasmic accumulation of $\alpha$-synuclein and ubiquitin in the nigral TH-positive neurons at four weeks after intrastriatal LPS. (A) Photomicrograph of double immunofluorescent labeling with antibodies against TH (red) and $\alpha$-synuclein (green) show that intrastriatal LPS mediates marked TH-positive cell loss in the substantia nigra ipsilateral to the injection. Scale bar: $100 \mu \mathrm{m}$. (B) High magnification images of the top photograph demonstrate that some of the spared TH-positive neurons have accumulated $\alpha$-synuclein in their cytoplasm (arrow heads). Scale bar: $20 \mu \mathrm{m}$. (C) Immunofluorescent staining displays ubiquitin accumulation in the cytoplasm of the nigral TH-positive neurons (arrow heads). Scale bar: $20 \mu \mathrm{m}$. (D-E) Increase of proteinase K-resistant $\alpha$-synuclein (D) or ubiquitin (E) in the substantia nigra after LPS injection. Scale bar: $50 \mu \mathrm{m}$. doi:10.1371/journal.pone.0005482.g003

\section{Intracytoplasmic accumulations of $\alpha$-synuclein and ubiquitin}

One of the pathological hallmarks of PD is the formation of Lewy bodies, a proteinaceous cytoplasmic inclusion containing $\alpha$ synuclein and ubiquitin [37]. Thus, we double immunostained midbrain sections with antibodies to TH and $\alpha$-synuclein, or TH and ubiquitin to assess intracytoplasmic accumulations of these proteins following striatal inflammation. Fluorescent microscopic analysis of dual-stained $\mathrm{TH}$ and $\boldsymbol{\alpha}$-synuclein demonstrated smeared immunostaining of $\alpha$-synuclein in the neuronal cytoplasm of the vehicle-injected side. However, an accumulation of $\alpha$ synuclein in the cytoplasm of surviving TH-positive neurons was observed in the substantia nigra ipsilateral to the LPS injection (Fig. 3A,B). Immunofluorescent staining also revealed that ubiquitin accumulates in the spared dopaminergic neurons following intrastriatal LPS injections (Fig. 3C). In an attempt to visualize insoluble form of $\alpha$-synuclein or ubiquitin, we pretreated midbrain sections with proteinase $\mathrm{K}$, and then immunostained with antibodies against $\boldsymbol{\alpha}$-synuclein or ubiquitin. Intrastriatal LPS injection increased the immunoreactivity of $\alpha$-synuclein (Fig. 3D) and ubiquitin (Fig. 3E) in the ipsilateral nigral neurons.

\section{Behavioral impairments}

Amphetamine-induced rotational behavior was analyzed to assess the unilateral degeneration of the presynaptic dopaminergic neuron terminals. Vehicle-injected rats did not show any significant bias in turning behavior after receiving an amphetamine injection. We did not observe the drug-induced rotational behavior at one week and two weeks after LPS challenge (data not shown). However, intrastriatal LPS caused a marked ipsilateral rotational behavior toward the lesioned side $(p=0.031)$ upon amphetamine challenge, four weeks after LPS injections (Fig. 4A).
The cylinder test was carried out at one week, two weeks, and four weeks post- LPS injection to assess an independent forelimb touch of animals to support their body against a cylinder wall [34]. Pronounced asymmetric forelimb use was developed by unilateral intrastriatal LPS injection, but not by vehicle injections. Asymmetric forelimb use was significantly increased at all three time points $(p=0.004, p=0.005$, and $p=0.013$ respectively) (Fig. 4B).
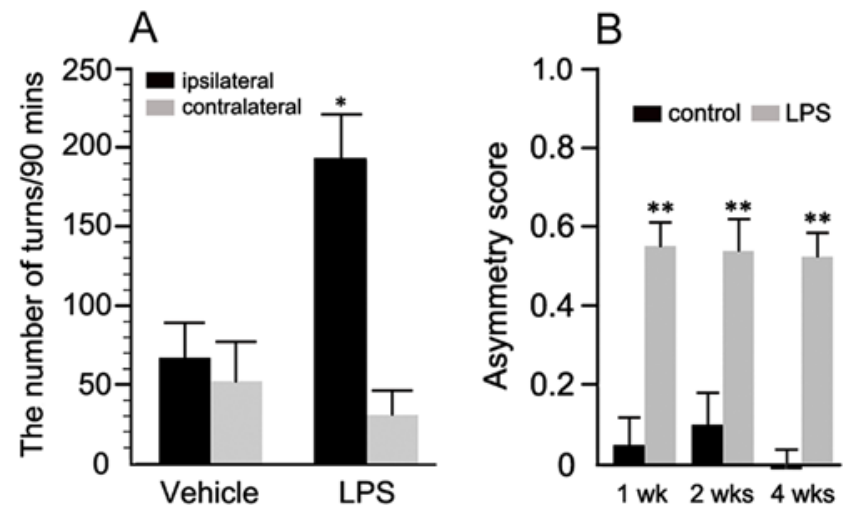

Figure 4. Behavioral deficits following intrastriatal LPS. (A) Ipsilateral rotational behavior in the unilateral LPS-injected animals is significantly increased relative to vehicle-treated animals when amphetamine was administered $\left(n=5-6 /\right.$ group; $\left.{ }^{*} p<0.05\right)$. (B) The cylinder test revealed that asymmetric forelimb use is increased significantly after intrastriatal LPS and was sustained for four weeks ( $n=5$-6/group; ** $p<0.01$ ).

doi:10.1371/journal.pone.0005482.g004 

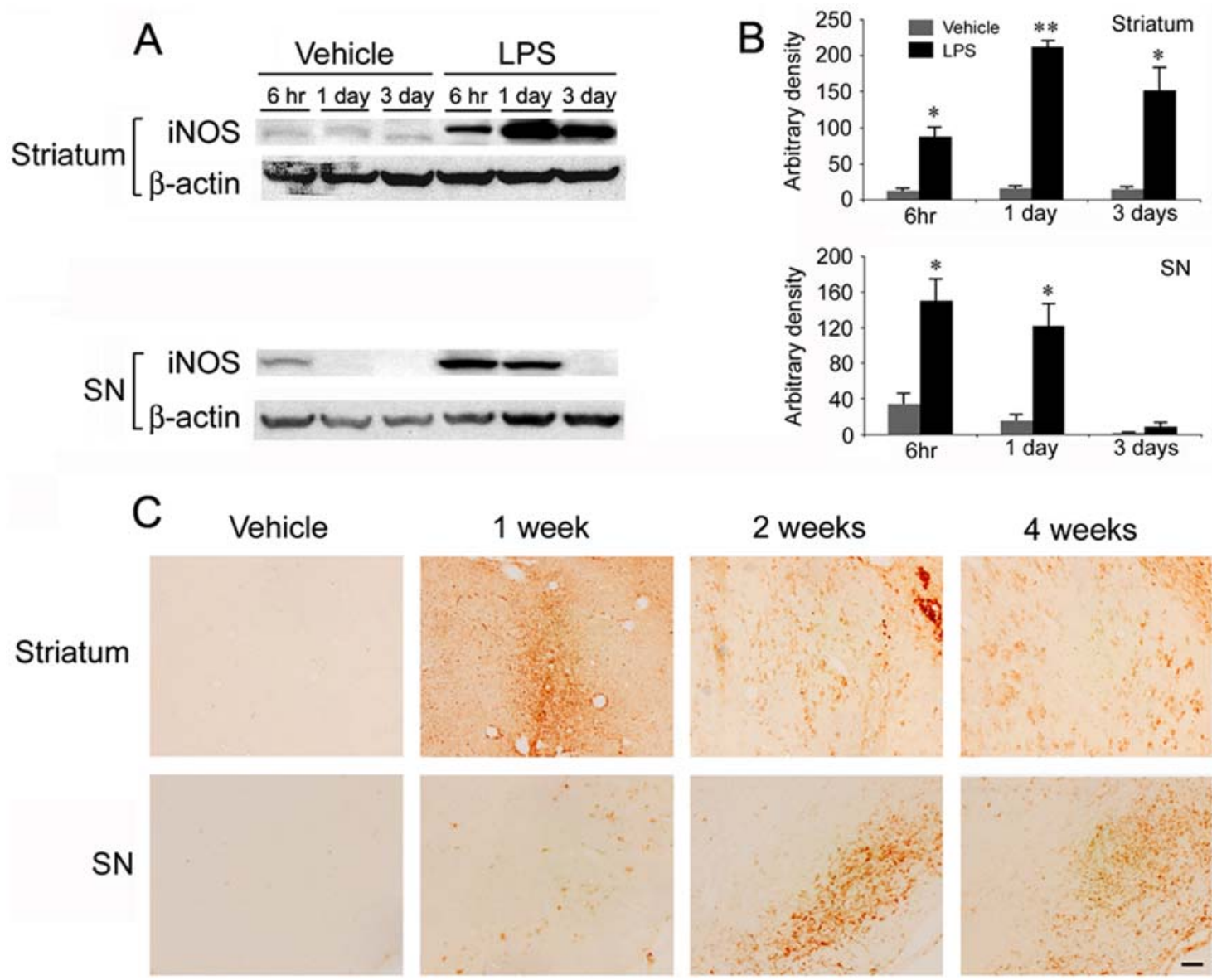

Figure 5. Microglial activation and elevation of iNOS expression in both the substantia nigra and striatum following intrastriatal LPS injection. The increased iNOS expression occurs at $6 \mathrm{hr}$ post LPS injection, which is sustained for three days in the striatum, and one day in the substantia nigra ( $n=3$ /group, ${ }^{*} p<0.05$, ${ }^{* *} p<0.01$ vs. control) $(A, B)$. The OX-6 immunoreactivity in the LPS-injected striatum is markedly increased one week after LPS injections compared to control or the naïve side, and immunoreactivity of OX-6 gradually decreased over time. However, the immunoreactivity is still positive four weeks after LPS (C, top). OX-6-positive microglia appear in the substantia nigra one week after intrastriatal LPS injection, and the immunoreactivity peaks at two weeks and then decreases to some extent at four weeks (C, bottom). Scale bar: $50 \mu \mathrm{m}$. doi:10.1371/journal.pone.0005482.g005

\section{Neuroinflammation in the nigrostriatal pathway after intrastriatal LPS injection}

We characterized neuroinflammation by immunostaining of MHC class II (OX-6), a marker for activated microglia and by measuring the transcriptional induction of proinflammatory cytokine genes via the RNase protection assay following intrastriatal LPS injection. We also performed western blot analysis to see the temporal and regional patterns of iNOS expression after LPS challenge.

Increased iNOS expression began to be detected $6 \mathrm{hr}$ after LPS challenge in both the substantia nigra $(p=0.035)$ and striatum $(p=0.021)$ (Fig. 5A,B). The increased nigral iNOS immunoreactivity was gradually reduced as time passed, and returned to control level at three days $(\mathrm{p}=0.042$ at one day, and $p=0.265$ at three days), while the increase in striatal iNOS expression reached a peak at one day and was still prominent at three days $(p=0.007$ at one day, and $p=0.027$ at three days $)$ (Fig. 5A,B).

Injection of LPS into the striatum markedly increased the number of OX-6-positive microglia in the striatum at seven days post injections, and the increased immunoreactivity remained elevated for four weeks (Fig. 5C, top panel). Immunoreactivity for OX-6 began to appear in the ipsilateral substantia nigra one week after LPS injection, peaked at two weeks, and was still prominent four weeks after LPS (Fig. 5C, bottom panel).

Significant transcriptional increases of IL- $1 \alpha(p=0.006)$, TNF$\alpha(p=0.007)$, IL-1 $\beta \quad(p=0.003)$, and IL-6 $(p=0.001)$ were measured in the LPS-treated striatum three hours after LPS injection. The level of TNF- $\alpha$ mRNA remained significantly elevated for up to one day $(p=0.043)$ and IL-1 $\beta$ for three days $(p=0.027)$ after LPS (Fig. S3 a,b). Interestingly, there was a significant increase in the mRNA expression of IL-1 $\beta$ $\left(p=3 \times 10^{-4}\right)$ and IL-6 $(p=0.002)$ in the ipsilateral substantia nigra, as early as three hours (Fig. S3 c,d).

\section{Mitochondrial dysfunction}

Intrastriatal LPS induced a significant decrease in nigral $(81.3 \%$ of control, $p=0.045)$ and striatal $(85.3 \%$ of control, $p=0.028)$ mitochondria state III respiration (the ability to phosphorylate ADP into ATP), which was efficiently prevented by treatment of L-NIL (Fig. 6A,B). When using the substrates pyruvate and malate, in the presence of carbonyl cyanide 4-trifluoromethoxy phenylhydrazone for maximum (state V) respiration, LPS induced a significant decrease in complex I activity of both nigral $(80.9 \%$ of control, $\mathrm{p}=0.035)$ and striatal $(81.2 \%$ of control, $\mathrm{p}=0.032)$ mitochondria. This impairment in complex I-driven state $\mathrm{V}$ 
A

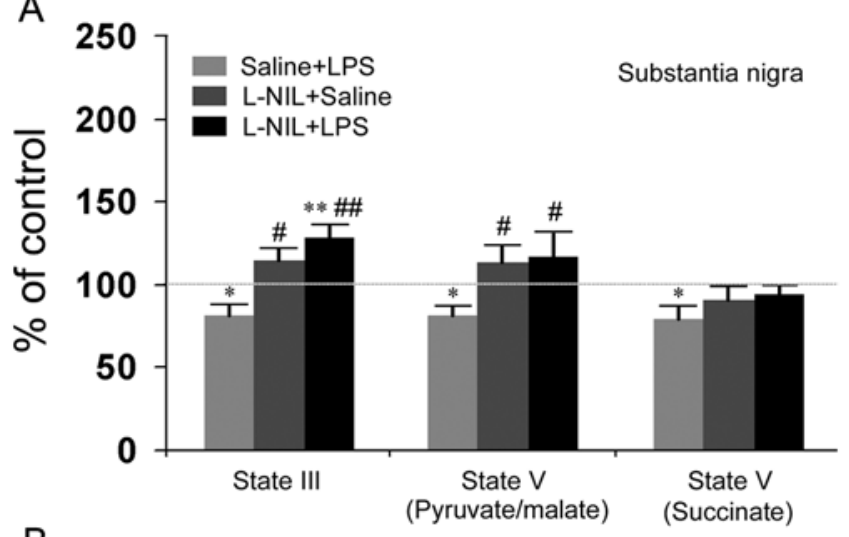

B

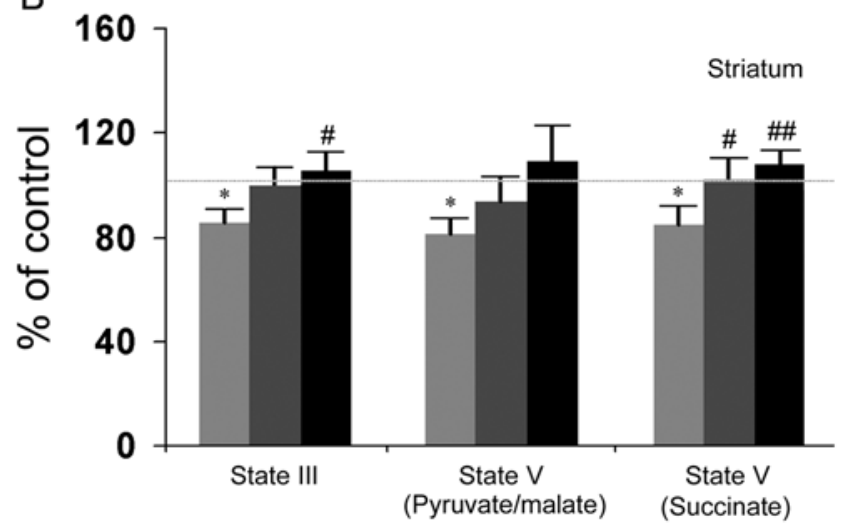

Figure 6. LPS impairs nigrostriatal mitochondria respiration. (A) Functional impairment occurs in the nigral mitochondria as LPS significantly decreases state III and state $\mathrm{V}$ respiration when driven by both complex I and complex II substrates. Treatment of L-NIL, an iNOS inhibitor prevents LPS-induced mitochondrial dysfunction ( $n=6 /$ group; ${ }^{*} p<0.05$, ** $p<0.01$ vs. saline+saline, \# $p<0.05$, \#\# $p<0.01$ vs. saline+LPS). (B) It appears that there is a significant decrease in state III and state $\mathrm{V}$ respiration of striatal mitochondria when driven by both complex I and complex II substrates in the striatum ipsilateral to LPS injection. L-NIL efficiently blocks the neuroinflammation-mediated defect in striatal mitochondrial respiration $\left(n=9 /\right.$ group; ${ }^{*} p<0.05$, ** $\mathrm{p}<0.01$ vs. Saline+Saline, \# $\mathrm{p}<0.05$, \#\# $\mathrm{p}<0.01$ vs. Saline+LPS). doi:10.1371/journal.pone.0005482.g006

respiration was blocked by treatment with the iNOS inhibitor. When utilizing succinate, the substrate for complex II driven respiration, state $\mathrm{V}$ respiration was also significantly reduced in the substantia nigra $(79.0 \%$ of control, $\mathrm{p}=0.021)$ and striatum $(84.6 \%$ of control, $p=0.024$ ) ipsilateral to LPS challenge (Fig. 6A,B). The reduction in complex II-driven state $\mathrm{V}$ respiration was prevented by treatment with L-NIL. This mitochondrial malfunction was not as marked as that of a previous study [30], which may reflect washoff or severely damaged mitochondria during the isolation, as a Ficoll gradient method was used here.

\section{S-nitrosylation and nitration of mitochondrial complex I}

To determine if S-nitrosylation or nitration of mitochondrial proteins is involved in the neuroinflammation-mediated mitochondrial dysfunction, we analyzed the nitration and/or Snitrosylation level of mitochondrial complex I. Mitochondrial function can be compromised by extensive nitration or $\mathrm{S}$ nitrosylation of mitochondrial complex I [18]. A significant elevated nitration of complex I $(p=0.038)$ occurred in the substantia nigra and striatum three days after LPS challenge
(Fig. 7A,B). Treatment with L-NIL efficiently prevented the increased protein nitration. LPS injection significantly increased in S-nitrosylation of complex $\mathrm{I}$ in the substantia nigra, which was blocked by L-NIL injection (Fig. 7C,D).

\section{Discussion}

Neuroinflammation, mitochondrial dysfunction and selective loss of dopaminergic neurons have been consistently observed in the substantia nigra of PD patients $[1,6,20]$. However, the correlation among these three in PD remains unclear. Here, we showed that neuroinflammation is able to mediate mitochondrial impairment by S-nitrosylation/nitration of mitochondrial proteins. This is followed by progressive dopaminergic neurodegeneration in the nigrostriatal system.

The loss of the dopaminergic neurons might be attributable to the intrinsic sensitivity of dopaminergic neurons to compromised mitochondrial function. Greenamyre and colleagues reported that systemic administration of a complex I inhibitor, rotenone to rats produced a selective degeneration of dopaminergic neurons in the substantia nigra [23]. Consistent with their report, we recently found that trichloroethylene causes selective loss of the nigral dopaminergic neurons in rats via complex I inhibition, and long term exposure to the chemical may be related to the development of parkinsonism in a group of factory workers [38]. Furthermore, mitochondrial dysfunction alone is sufficient to initiate parkinsonism in conditional knock-out mice by disrupting the gene for mitochondrial transcription factor $\mathrm{A}$ in the nigral dopamine neurons [39]. These observations are in agreement with the fact that rare familial forms of PD are related to mutations in the gene encoding PINK1 or DJ1, which both regulate mitochondrial function [40]. Thus, it can be questioned, what makes the nigral dopaminergic neurons substantially susceptible to mitochondrial dysfunction. A recent study showed that nigral dopamine neurons unusually rely on L-type voltage-gated calcium ion channels for basal activity, and the reliance increases with age [41]. The high dependency on the calcium channel leads to sustained elevation in cytosolic calcium concentration, which enhances mitochondrial respiration, reactive oxygen species generation, and ATP demand [42-44]. Therefore, the nigral dopaminergic neurons can be devastated by mitochondrial insults, which are tolerable to the other populations of neurons.

The implication of NO in PD pathogenesis is supported by the observations that iNOS expression is upregulated in activated microglia $[11,45]$, the immunoreactivity of nitrated $\alpha$-synuclein is prominently positive in the Lewy bodies [46,47], and several enzymes including parkin, peroxiredoxin-2, and protein-disulphide isomerase lose their function by S-nitrosylation in PD brains [48-50]. Although association of NO with mitochondrial dysfunction in PD is unclear, NO and its metabolite, peroxynitrite can compromise mitochondrial biomolecules to induce impairment in mitochondrial respiration. It has been well known that NO reversibly inhibit cytochrome c oxidase activity and depletes mitochondrial antioxidants such as glutathione [51]. Importantly, peroxynitrite and high level of NO irreversibly inactivates mitochondrial complex I activity by tyrosine nitration, Snitrosylation, oxidation of residues and damage of iron sulfur center $[18,52]$. These notions are in agreement with our results that intrastriatal LPS injection leads to complex I nitration and Snitrosylation, and is accompanied decrease in mitochondrial respiration rate. This evidence strongly supports the hypothesis that excessive production of $\mathrm{NO}$ in the brain might contribute to mitochondrial dysfunction and subsequent neuronal energy deficiency observed in PD. 
A

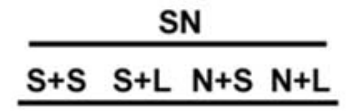

anti-3-NT

anti-complex I

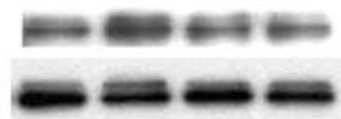

SN

S+S S+L N+S N+L

anti-3-NT

anti-complex I

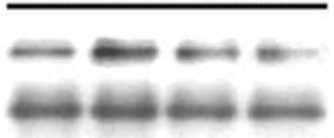

Striatum

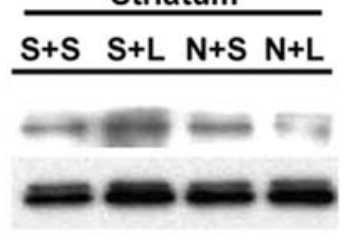

Striatum

$\mathrm{S}+\mathrm{S} \mathrm{S}+\mathrm{L} \mathrm{N}+\mathrm{S} \mathrm{N}+\mathrm{L}$
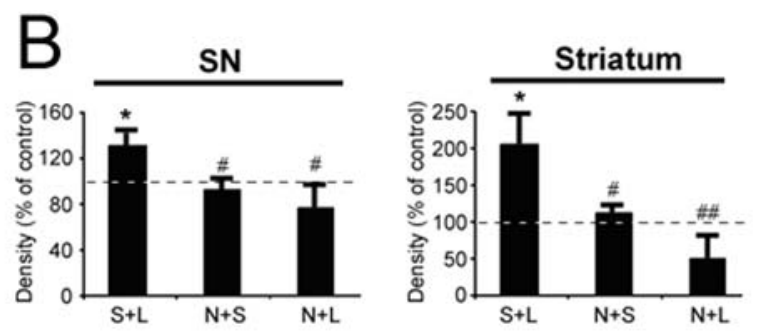

D

SN

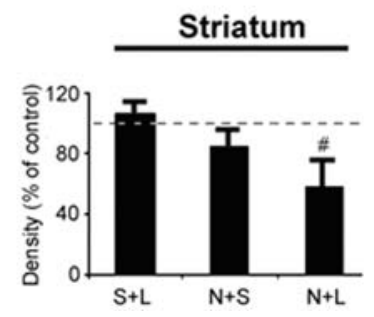

Figure 7. Nitration/S-nitrosylation of mitochondrial complex I after intrastriatal LPS. Intrastriatal LPS injection increases 3-nitrotyrosine (3NT) level in complex I three days after LPS injection. Treatment with L-NIL appears to prevent the LPS-induced elevation of mitochondrial protein nitration $(A, B)$. Isolated nigral and striatal mitochondria complex I proteins have an increase in S-nitrosylation three days after LPS injection. L-NIL treatment appears to prevent the LPS-induced increase of S-nitrosylation (C,D). S+S: saline treated and saline injected; S+L: saline-treated and LPSinjected; N+S: L-NIL-treated and saline-injected; and N+L: L-NIL-treated and LPS-injected; SN: substantia nigra $\left(n=4 / g r o u p ;{ }^{*} p<0.05\right.$ vs. S+S, and \# $\mathrm{p}<0.05, \mathrm{p}<0.01$ vs. S+L)

doi:10.1371/journal.pone.0005482.g007

Inflammatory activation of microglia is related to increases in release of glutamate and consequent excitotoxicity [53]. It has been reported that neuronal NOS (nNOS)-derived NO accounts, at least in part, for glutamate-induced excitotoxicity [54]. We found that activated microglia appears one week after LPS challenge, and the microgliosis were sustained for four weeks in the substantia nigra. In addition, we observed that treatment of L$\mathrm{N}(\mathrm{G})$-nitro-arginine, an inhibitor of nNOS, was also neuroprotective against intrastriatal LPS in mice, suggesting the possible contribution of nNOS to the dopamine neurodegeneration [55]. This is further supported by our observation of cytoplasmic accumulation of $\alpha$-synuclein and ubiquitin in the nigral dopamineproducing neurons, as extensive nitrosative/oxidative stress might cause impairment of the ubiquitin proteasome system resulting in accumulation of misfolded proteins [50,56]. Support to the notion comes from the result that the accumulation of $\alpha$-synuclein was attenuated by treatment with L-NIL (unpublished data). Hence, our results might be relevant for the molecular pathway for Lewy body formation and PD pathogenesis.

It is interesting that loss of a relatively small population of nigral dopamine neurons caused a decrease in the striatal dopamine level and behavioral impairment in the LPS-treated animals. The behavioral deficit began even at early time-point like one week following LPS injection. This phenomenon may suggest that the striatal inflammation antagonizes the function of the dopaminergic nigrostriatal pathway via altering synthesis and/or release of dopamine, or by modulating dopamine-mediated signal transduction before a significant demise of the dopaminergic neurons occurs. In support of this suggestion, it was shown that MPTP treatment induces striatal $\mathrm{TH}$ nitration, which was related to inactivation of the enzyme and a subsequent greater decline in dopamine level, compared to the loss of the dopaminergic neurons [57]. In addition, direct injection of proinflammatory mediators into the striatum such as prostaglandin D2 and the thromboxane A2 agonist induced impairment of motor behavior; although, the specific mechanism was not elucidated $[58,59]$. Therefore, further study is required to fully illuminate the mechanism by which neurochemical alterations and behavioral deficit occur following intrastriatal LPS injections.

Taken together, results of the present study provide strong support to the hypothesis that neuroinflammation may significantly contribute to PD pathogenesis. This animal model may be useful for studying neuroinflammatory mechanisms by which nigral dopamine neurons degenerate in PD.

\section{Supporting Information}

Figure S1 LPS-induced neuroinflammation significantly decreases TH-positive fiber density in the striatum four weeks after LPS challenge ( $\mathrm{n}=5$ /group, $* * * \mathrm{p}<0.001$ vs. control).

Found at: doi:10.1371/journal.pone.0005482.s001 (2.50 MB TIF)

Figure S2 Decrease in striatal serotonin level following LPS injection. HPLC analysis reveals that intrastriatal LPS injection significantly reduces serotonin level, but not its metabolite 5-HIAA resulting in marked elevation of the turnover ratio (5-HIAA/ serotonin) ( $\mathrm{n}=7$ /group, $* * * \mathrm{p}<0.001$ vs. control).

Found at: doi:10.1371/journal.pone.0005482.s002 (2.59 MB TIF)

Figure S3 The elevated mRNA levels of proinflammatory cytokines in the striatum and substantia nigra. (A,B) RPA shows that the mRNA of the proinflammatory cytokines TNF-alpha, and IL-1beta begin to significantly increase three hours after LPS injections in the striatum, compared to the naïve or vehicle treated striatum, which is sustained for up to three days following LPS. The elevated mRNA levels of IL-lalpha and IL-6 occur three hours after LPS injection in the striatum, compared to the naïve or vehicle treated striatum, and return to control level at six hours following LPS ( $\mathrm{n}=4$ /group; * $\mathrm{p}<0.05$, ** $\mathrm{p}<0.01$ ). (C,D) RPA demonstrates that mRNA levels of IL-1beta and IL-6 are significantly increased in the substantia nigra three hours after LPS injection and remain significantly increased for six hours, when compared with the naïve or vehicle treated substantia nigra. IL-lalpah and TNF-alpha only show a trend to be increased at the three hour time point $(\mathrm{n}=4 /$ group; $* \mathrm{p}<0.05, * * \mathrm{p}<0.01)$. 
Found at: doi:10.1371/journal.pone.0005482.s003 (5.90 MB TIF)

\section{Acknowledgments}

We are grateful to Ms. Laura Peters for the excellent technical support of HPLC analysis.

\section{References}

1. McGeer PL, Itagaki S, Boyes BE, McGeer EG (1988) Reactive microglia are positive for HLA-DR in the substantia nigra of Parkinson's and Alzheimer's disease brains. Neurology 38: 1285-1291.

2. Batchelor PE, Liberatore GT, Wong JY, Porritt MJ, Frerichs F, et al. (1999) Activated macrophages and microglia induce dopaminergic sprouting in the injured striatum and express brain-derived neurotrophic factor and glial cell linederived neurotrophic factor. J Neurosci 19: 1708-1716.

3. Banati RB, Gehrmann J, Schubert P, Kreutzberg GW (1993) Cytotoxicity of microglia. Glia 7: 111-118.

4. Gehrmann J, Banati RB, Wiessner C, Hossmann KA, Kreutzberg GW (1995) Reactive microglia in cerebral ischaemia: an early mediator of tissue damage? Neuropathol Appl Neurobiol 21: 277-289.

5. Banati RB, Daniel SE, Blunt SB (1998) Glial pathology but absence of apoptotic nigral neurons in long-standing Parkinson's disease. Mov Disord 13: 221-227.

6. Block ML, Zecca L, Hong JS (2007) Microglia-mediated neurotoxicity: uncovering the molecular mechanisms. Nat Rev Neurosci 8: 57-69.

7. Langston JW, Forno LS, Tetrud J, Reeves AG, Kaplan JA, et al. (1999) Evidence of active nerve cell degeneration in the substantia nigra of humans years after 1methyl-4-phenyl-1,2,3,6-tetrahydropyridine exposure. Ann Neurol 46: 598-605.

8. McGeer PL, Schwab C, Parent A, Doudet D (2003) Presence of reactive microglia in monkey substantia nigra years after 1-methyl-4-phenyl-1,2,3,6tetrahydropyridine administration. Ann Neurol 54: 599-604.

9. Ouchi Y, Yoshikawa E, Sekine Y, Futatsubashi M, Kanno T, et al. (2005) Microglial activation and dopamine terminal loss in early Parkinson's disease. Ann Neurol 57: 168-175.

10. Chen H, Jacobs E, Schwarzschild MA, McCullough ML, Calle EE, et al. (2005) Nonsteroidal antiinflammatory drug use and the risk for Parkinson's disease. Ann Neurol 58: 963-967

11. Knott C, Stern G, Wilkin GP (2000) Inflammatory regulators in Parkinson's disease: iNOS, lipocortin-1, and cyclooxygenases-1 and -2. Mol Cell Neurosci 16: $724-739$.

12. Arimoto T, Bing G (2003) Up-regulation of inducible nitric oxide synthase in the substantia nigra by lipopolysaccharide causes microglial activation and neurodegeneration. Neurobiol Dis 12: 35-45.

13. Liberatore GT, Jackson-Lewis V, Vukosavic S, Mandir AS, Vila M, et al. (1999) Inducible nitric oxide synthase stimulates dopaminergic neurodegeneration in the MPTP model of Parkinson disease. Nat Med 5: 1403-1409.

14. Teismann P, Tieu K, Choi DK, Wu DC, Naini A, et al. (2003) Cyclooxygenase2 is instrumental in Parkinson's disease neurodegeneration. Proc Natl Acad Sci U S A 100: 5473-5478.

15. Bal-Price A, Brown GC (2001) Inflammatory neurodegeneration mediated by nitric oxide from activated glia-inhibiting neuronal respiration, causing glutamate release and excitotoxicity. J Neurosci 21: 6480-6491.

16. Radi R (2004) Nitric oxide, oxidants, and protein tyrosine nitration. Proc Natl Acad Sci U S A 101: 4003-4008.

17. Tangpong J, Cole MP, Sultana R, Estus S, Vore M, et al. (2007) Adriamycinmediated nitration of manganese superoxide dismutase in the central nervous system: insight into the mechanism of chemobrain. J Neurochem 100: 191-201.

18. Brown GC, Borutaite V (2004) Inhibition of mitochondrial respiratory complex I by nitric oxide, peroxynitrite and S-nitrosothiols. Biochim Biophys Acta 1658: 44- 49 .

19. Schapira AH, Cooper JM, Dexter D, Jenner P, Clark JB, et al. (1989) Mitochondrial complex I deficiency in Parkinson's disease. Lancet 1: 1269.

20. Schapira AH (2007) Mitochondrial dysfunction in Parkinson's disease. Cell Death Differ 14: 1261-1266.

21. Langston JW, Ballard P, Tetrud JW, Irwin I (1983) Chronic Parkinsonism in humans due to a product of meperidine-analog synthesis. Science 219: 979-980.

22. Langston JW, Langston EB, Irwin I (1984) MPTP-induced parkinsonism in human and non-human primates--clinical and experimental aspects. Acta Neurol Scand Suppl 100: 49-54.

23. Betarbet R, Sherer TB, MacKenzie G, Garcia-Osuna M, Panov AV, et al. (2000) Chronic systemic pesticide exposure reproduces features of Parkinson's disease. Nat Neurosci 3: 1301-1306.

24. Antunes F, Han D, Rettori D, Cadenas E (2002) Mitochondrial damage by nitric oxide is potentiated by dopamine in PC12 cells. Biochim Biophys Acta 1556: 233-238.

25. Cohen G, Kesler N (1999) Monoamine oxidase and mitochondrial respiration. J Neurochem 73: 2310-2315.

26. Herrera AJ, Castano A, Venero JL, Cano J, Machado A (2000) The single intranigral injection of LPS as a new model for studying the selective effects of inflammatory reactions on dopaminergic system. Neurobiol Dis 7: 429-447.

\section{Author Contributions}

Conceived and designed the experiments: DYG RLH HCK GB. Performed the experiments: DYG ML WAC JDP EJS. Analyzed the data: DYC RLH JDP EJS DMG GB. Contributed reagents/materials/analysis tools: PGS. Wrote the paper: DYC RLH. Quality control: DMG.

27. Hsieh PF, Chia LG, Ni DR, Cheng LJ, Ho YP, et al. (2002) Behavior, neurochemistry and histology after intranigral lipopolysaccharide injection. Neuroreport 13: 277-280.

28. Arimoto T, Choi DY, Lu X, Liu M, Nguyen XV, et al. (2007) Interleukin-10 protects against inflammation-mediated degeneration of dopaminergic neurons in substantia nigra. Neurobiol Aging 28: 894-906.

29. Zhang J, Stanton DM, Nguven XV, Liu M, Zhang Z, et al. (2005) Intrapallidal lipopolysaccharide injection increases iron and ferritin levels in glia of the rat substantia nigra and induces locomotor deficits. Neuroscience 135: 829-838.

30. Hunter RL, Dragicevic N, Seifert K, Choi DY, Liu M, et al. (2007) Inflammation induces mitochondrial dysfunction and dopaminergic neurodegeneration in the nigrostriatal system. J Neurochem 100: 1375-1386.

31. Liu M, Hunter R, Nguyen XV, Kim HC, Bing G (2008) Microsomal epoxide hydrolase deletion enhances tyrosine hydroxylase phosphorylation in mice after MPTP treatment. J Neurosci Res 86: 2792-2801.

32. Georgievska B, Carlsson T, Lacar B, Winkler C, Kirik D (2004) Dissociation between short-term increased graft survival and long-term functional improvements in Parkinsonian rats overexpressing glial cell line-derived neurotrophic factor. Eur J Neurosci 20: 3121-3130.

33. Cass WA, Harned ME, Peters LE, Nath A, Maragos WF (2003) HIV-1 protein Tat potentiation of methamphetamine-induced decreases in evoked overflow of dopamine in the striatum of the rat. Brain Res 984: 133-142.

34. Lundblad M, Andersson M, Winkler C, Kirik D, Wierup N, et al. (2002) Pharmacological validation of behavioural measures of akinesia and dyskinesia in a rat model of Parkinson's disease. Eur J Neurosci 15: 120-132.

35. Brown MR, Sullivan PG, Geddes JW (2006) Synaptic mitochondria are more susceptible to Ca2+overload than nonsynaptic mitochondria. J Biol Chem 281: 11658-11668.

36. Zigmond MJ, Abercrombie ED, Berger TW, Grace AA, Stricker EM (1990) Compensations after lesions of central dopaminergic neurons: some clinical and basic implications. Trends Neurosci 13: 290-296.

37. Moore DJ, West AB, Dawson VL, Dawson TM (2005) Molecular pathophysiology of Parkinson's disease. Annu Rev Neurosci 28: 57-87.

38. Gash DM, Rutland K, Hudson NL, Sullivan PG, Bing G, et al. (2008) Trichloroethylene: Parkinsonism and complex 1 mitochondrial neurotoxicity. Ann Neurol 63: 184-192

39. Ekstrand MI, Terzioglu M, Galter D, Zhu S, Hofstetter C, et al. (2007) Progressive parkinsonism in mice with respiratory-chain-deficient dopamine neurons. Proc Natl Acad Sci U S A 104: 1325-1330.

40. Schapira AH (2006) Mitochondrial disease. Lancet 368: 70-82.

41. Chan CS, Guzman JN, Ilijic E, Mercer JN, Rick C, et al. (2007) 'Rejuvenation' protects neurons in mouse models of Parkinson's disease. Nature 447: 1081-1086.

42. Dawson TM, Dawson VL (2003) Molecular pathways of neurodegeneration in Parkinson's disease. Science 302: 819-822.

43. Dauer W, Przedborski S (2003) Parkinson's disease: mechanisms and models. Neuron 39: 889-909.

44. Orrenius S, Zhivotovsky B, Nicotera P (2003) Regulation of cell death: the calcium-apoptosis link. Nat Rev Mol Cell Biol 4: 552-565.

45. Hunot S, Boissiere F, Faucheux B, Brugg B, Mouatt-Prigent A, et al. (1996) Nitric oxide synthase and neuronal vulnerability in Parkinson's disease. Neuroscience 72: 355-363.

46. Duda JE, Giasson BI, Chen Q, Gur TL, Hurtig HI, et al. (2000) Widespread nitration of pathological inclusions in neurodegenerative synucleinopathies. Am J Pathol 157: 1439-1445.

47. Giasson BI, Duda JE, Murray IV, Chen Q, Souza JM, et al. (2000) Oxidative damage linked to neurodegeneration by selective alpha-synuclein nitration in synucleinopathy lesions. Science 290: 985-989.

48. Fang J, Nakamura T, Cho DH, Gu Z, Lipton SA (2007) S-nitrosylation of peroxiredoxin 2 promotes oxidative stress-induced neuronal cell death in Parkinson's disease. Proc Natl Acad Sci U S A 104: 18742-18747.

49. Chung KK, Thomas B, Li X, Pletnikova O, Troncoso JC, et al. (2004) Snitrosylation of parkin regulates ubiquitination and compromises parkin's protective function. Science 304: 1328-1331.

50. Uehara T, Nakamura T, Yao D, Shi ZQ, Gu Z, et al. (2006) S-nitrosylated protein-disulphide isomerase links protein misfolding to neurodegeneration. Nature 441: 513-517.

51. Moncada S, Bolanos JP (2006) Nitric oxide, cell bioenergetics and neurodegeneration. J Neurochem 97: 1676-1689.

52. Clementi E, Brown GC, Feelisch M, Moncada S (1998) Persistent inhibition of cell respiration by nitric oxide: crucial role of S-nitrosylation of mitochondrial complex I and protective action of glutathione. Proc Natl Acad Sci U S A 95: 7631-7636. 
53. Barger SW, Basile AS (2001) Activation of microglia by secreted amyloid precursor protein evokes release of glutamate by cystine exchange and attenuates synaptic function. J Neurochem 76: 846-854.

54. Dawson VL, Dawson TM, London ED, Bredt DS, Snyder SH (1991) Nitric oxide mediates glutamate neurotoxicity in primary cortical cultures. Proc Natl Acad Sci U S A 88: 6368-6371.

55. Hunter RL, Cheng B, Choi DY, Liu M, Liu S, et al. (2009) Intrastriatal lipopolysaccharide injection induces Parkinsonism in C57/B6 mice J Neurosci Res.

56. McNaught KS, Olanow CW, Halliwell B, Isacson O, Jenner P (2001) Failure of the ubiquitin-proteasome system in Parkinson's disease. Nat Rev Neurosci 2: 589-594.
57. Ara J, Przedborski S, Naini AB, Jackson-Lewis V, Trifiletti RR, et al. (1998) Inactivation of tyrosine hydroxylase by nitration following exposure to peroxynitrite and 1-methyl-4-phenyl-1,2,3,6-tetrahydropyridine (MPTP). Proc Natl Acad Sci U S A 95: 7659-7663.

58. Costall B, Holmes SW, Kelly ME, Naylor RJ (1985) Modification of dyskinesias following the intrastriatal injection of prostaglandins in the rodent. Br J Pharmacol 85: 943-949.

59. Yagami T, Takahara Y, Ishibashi C, Sakaguchi G, Itoh N, et al. (2004) Amyloid beta protein impairs motor function via thromboxane A2 in the rat striatum. Neurobiol Dis 16: 481-489. 